\title{
Dependence of Cardiac Systolic Function on Elevated Fatty Acid Availability in Obese, Insulin-Resistant Rats.
}

Wayne Smith, $\mathrm{PhD}^{1,2}$, Gavin R. Norton, $\mathrm{PhD}, \mathrm{MD}^{3}$, Angela J. Woodiwiss $\mathrm{PhD}^{3}$, Amanda Lochner $\mathrm{DSc}^{1}$, Eugene F. du Toit, $\mathrm{PhD}^{4}$.

${ }^{1}$ Department of Biomedical Sciences, Faculty of Health Sciences, University of Stellenbosch, PO BOX 19063, Tygerberg 7505, South Africa, ${ }^{2}$ Hypertension in Africa Research Team (HART), North West University, Private Bag X6001, Potchefstroom 2520, South Africa; ${ }^{3}$ Cardiovascular Pathophysiology and Genomics Research Unit, School of Physiology, Faculty of Health Sciences, University of the Witwatersrand, 7 York Road, Parktown, 2193, Johannesburg, South Africa and ${ }^{4}$ The School of Medical Science, Griffith University, Gold Coast Campus, Southport QLD 4217, Australia.

WS:22945717@nwu.ac.za; GRN:Gavin.Norton@wits.ac.za;

AJW: Angela.Woodiwiss@wits.ac.za; AL: alo@sun.ac.za;

EFdT: j.dutoit@griffith.edu.au

Abbreviated title: Obesity and cardiac systolic function.

Word count: 4301

Corresponding Author:

Prof. E.F. du Toit

School of Medical Science

Griffith University

Southport QLD 4217

AUSTRALIA

Ph: (+61) 755529728

Fax: (+61) 755528908

E-mail: j.dutoit@griffith.edu.au 


\section{Highlights}

- Ex vivo cardiac pump function of obese rats is compromised when glucose is the only substrate

- Fatty acids normalise cardiac pump function ex vivo in hearts from obese rats

- Elevated fatty acid levels are important to maintain normal LV systolic function in obesity 

Abbreviations
FA, fatty acid
$\mathrm{LV}$, left ventricular
LV Ees, the slope of the LV systolic pressure-volume relationship
LV En, left ventricular end systolic elastance
LVEDD, Left ventricular end diastolic diameter
LVESD; left ventricular end systolic diameter
FSend; endocardial fractional shortening
FSmid, midwall fractional shortening 


\begin{abstract}
Background: Clinical data advocating an adverse effect of obesity on left ventricular (LV) systolic function independent of comorbidities is controversial. We hypothesized that in obesity with pre-diabetic insulin resistance, circulating fatty acids (FA's) become a valuable fuel source in the maintenance of normal systolic function.

Methods: Male Wistar rats were fed a high caloric diet for 32 weeks to induce obesity. Myocardial LV systolic function was assessed using echocardiography and isolated heart preparations.
\end{abstract}

Results: Aortic output was reduced in obese rat hearts over a range of filling pressures (for example: $15 \mathrm{cmH}_{2} \mathrm{O}$, obese: $32.6 \pm 1.2 \mathrm{ml} / \mathrm{min}$ vs. control: $46.2 \pm 0.9 \mathrm{ml} / \mathrm{min}, \mathrm{p}<0.05$ ) when perfused with glucose alone. Similarly, the slope of the LV end-systolic pressurevolume relationship decreased, and there was a right shift in the LV end-systolic stressstrain relationship as determined in Langendorff perfused, isovolumic rat heart preparations in the presence of isoproterenol $\left(10^{-8} \mathrm{M}\right)\left(\mathrm{LV}\right.$ En, obese: $791 \pm 62 \mathrm{~g} / \mathrm{cm}^{2} \mathrm{vs}$. control: $\left.1186 \pm 74 \mathrm{~g} / \mathrm{cm}^{2}, \mathrm{p}<0.01\right)$. The addition of insulin to the perfusion buffer improved aortic output while the addition of FA's completely normalized aortic output. LV function was maintained in obese animals in vivo during an inotropic challenge.

Conclusions: Elevated circulating FA levels may be important to maintain myocardial systolic function in the initial stages of obesity and insulin resistance.

Key words: obesity, fatty acids, cardiac systolic function. 


\section{INTRODUCTION}

Obesity is an independent risk factor for heart failure. ${ }^{1}$ However, whether obesity promotes cardiac pump dysfunction through mechanisms unrelated to hypertension and diabetes is still uncertain. In this regard, clinical studies conducted with overweight to severely obese patients have demonstrated either augmented ${ }^{2,3}$ preserved $^{4-8}$ or reduced $^{9}$ left ventricular (LV) systolic function independent of diabetes and hypertension. Other authors additionally report reduced load independent measures of systolic function (subclinical systolic dysfunction) with preserved ejection fraction in similar participants. ${ }^{10,11}$ Data from animal studies also provide inconclusive observations regarding the effect of diet induced obesity on LV systolic function. ${ }^{12-19}$

One potential explanation for the conflicting data regarding the independent effect of obesity on cardiac systolic function is that obesity may indeed be associated with intrinsic myocardial alterations that impair contraction, but that at least in the early stages of obesity, compensatory myocardial changes may occur that enable myocardial function to be preserved. This is supported by the observation that the duration of especially morbid obesity is associated with a reduction in LV systolic function (LV fractional shortening). ${ }^{20}$ In line with this hypothesis, obesity is associated with increased circulating fatty acid (FA) concentrations ${ }^{21}$ and in most instances elevated rates of myocardial FA

oxidation. ${ }^{16,22}$ Since the myocardium relies less on glucose as a fuel source in the setting of obesity and insulin resistance, ${ }^{16,22}$ it is plausible that increased FA availability combined with enhanced FA oxidation becomes vital to help maintain myocardial mechanical function in hearts that are metabolically compromised. We therefore tested 
the hypothesis that elevated circulating FA's and insulin associated with obesity and insulin resistance may compensate for an impaired myocardial systolic function noted in obese, insulin-resistant (pre-diabetic) rats when glucose is present as the only fuel source. In order to test this hypothesis we studied cardiac mechanical function in a previously described animal model of obesity and insulin-resistance. ${ }^{12,18,23,24}$ In the present study cardiac systolic function was determined both in vivo and ex vivo. In the ex vivo studies, we assessed cardiac mechanical performance in the absence and in the presence of FA's and insulin at concentrations found in vivo in lean and obese rats.

\section{METHODS}

\section{Animals}

This study was conducted in accordance with the Principles of Laboratory Animal Care of the National Society for Medical Research and the Guide for the Care and use of Laboratory Animals of the National Academy of Sciences (NIH publication no 80-23, revised 1985). Sixty male Wistar rats weighing 200 \pm 10 grams received a standard rodent chow supplemented with sucrose and condensed milk for 32 weeks. The experimental diet is designed to induce obesity through hyperphagia with the experimental group ( $\mathrm{n}=31$ ) consuming $570 \pm 23 \mathrm{~kJ} /$ day as compared to the control group $(\mathrm{n}=29)$ that consumed $371 \pm 18 \mathrm{~kJ} /$ day. ${ }^{12,25}$ The compositions of the standard rat chow and the experimental diet used in this study have previously been reported. ${ }^{24}$ We have previously demonstrated that this experimental diet induces both obesity and insulin resistance after 16 weeks of consumption. ${ }^{23,26}$ Blood pressures were determined using a tail cuff method. $^{27}$ 


\section{Isolated, perfused heart preparations.}

To assess whether the absence of circulating FA's and insulin resulted in cardiac pump dysfunction, left ventricular systolic function was assessed ex vivo in 1) the isolated working heart model (as modified by Opie et $\mathrm{al}^{28}$ ) to ensure that systolic function was assessed in a heart preparation performing work and 2) in the isolated retrograde perfused, isovolumic, constant coronary flow heart preparations ${ }^{29}$ to account for the effects of load on systolic function; to assess intrinsic myocardial systolic function (see below); and to account for potential effects of obesity on coronary flow.

Rats were anesthetized with an intraperitoneal injection of pentobarbitone-sodium (60 $\mathrm{mg} / \mathrm{kg}$ ) and the hearts rapidly excised. The hearts were placed in ice-cold buffer before being transferred to a heart perfusion apparatus where they were perfused with a KrebsHenseleit buffer equilibrated with $95 \% \mathrm{O}_{2}$ and $5 \% \mathrm{CO}_{2}$ at $37^{\circ} \mathrm{C}(\mathrm{pH} 7.4)$ (in $\mathrm{mmol} / \mathrm{L}$ $\mathrm{NaCl} 118.0, \mathrm{KCl} 4.7, \mathrm{MgSO}_{4} .7 \mathrm{H}_{2} \mathrm{O} 1.2, \mathrm{CaCl}_{2}$ 1.25, $\mathrm{NaHCO}_{3} 25, \mathrm{KH}_{2} \mathrm{PO}_{4}$ 1.2, glucose 10 (Merck Pty.Ltd., Darmstadt, Germany)) at a pressure of $100 \mathrm{~cm} \mathrm{H}_{2} \mathrm{O}$. Retrograde perfusion was initiated within 45 seconds of excision of the heart.

The influence of FA's or insulin (or the combination of both) on LV systolic function was only assessed in working heart preparations. FA's (final concentration: $0.7 \mathrm{mM}$ for control and $1.5 \mathrm{mM}$ for obese rats) were pre-bound to $3 \%$ bovine serum albumin (Fraction $\mathrm{V}$, Roche, Germany) and added to the perfusion buffer as previously described. ${ }^{30} \mathrm{FA}$ 's in the bovine serum albumin contributed $0.3 \mathrm{mM}$ to the FA concentration in the buffer and the rest of the FA's were added in the form of palmitate (Sigma-Aldrich, St.Louis, MO). Insulin (humulin-N, Eli Lilly, South Africa) was added to the perfusion buffer to achieve concentrations of $30 \mu \mathrm{IU} / \mathrm{ml}$ for control hearts and $50 \mu \mathrm{IU} / \mathrm{ml}$ in obese rat hearts. These 
FA and insulin concentrations closely resemble the plasma concentrations we previously reported in control and obese rats following 16 weeks of feeding. ${ }^{23}$

Working heart model: For working heart perfusions the left atrium was cannulated and hearts were perfused in the working heart mode initially at a preload of 15 $\mathrm{cmH}_{2} \mathrm{O}$. The preload was subsequently increased at five minute intervals from 15 to 17.5 $\mathrm{cmH}_{2} \mathrm{O}$ and then to $20 \mathrm{cmH}_{2} \mathrm{O}$. Afterload was maintained at $100 \mathrm{cmH}_{2} \mathrm{O}$. Hearts perfused with glucose or glucose and insulin as the only substrate were paced at $330 \mathrm{bpm}$ with the stimulation voltage $10 \%$ above threshold via platinum wire electrodes attached to the left atrium and the apex of the heart. Hearts perfused in the presence of glucose and FA's were not paced. The heart rates from these un-paced hearts were however comparable between control and obese animals (see results section). Hearts with a rate below 200 bpm were excluded from the study $(\mathrm{n}=1)$. During FA and insulin perfusion, the perfusion buffer $(200 \mathrm{ml})$ was recirculated for the duration of the experiment. This was not the case when glucose was used as the sole substrate. Coronary flow and aortic output were documented. Systolic performance was assessed from aortic output determined at incremental filling pressures.

Isovolumic, retrograde perfusion model: These studies were performed in a separate group of rats from those used for the working heart preparations, using an approach previously described. ${ }^{31}$ Once hearts were mounted on the perfusion apparatus, the coronary flow rate was determined volumetrically and adjusted to achieve a constant flow of $12 \mathrm{ml} / \mathrm{min} / \mathrm{g}$ heart weight. The hearts were paced at $300 \mathrm{bpm}$ as described above. LV developed pressure was determined by use of a water-filled balloon-tipped cannula coupled to a pressure transducer inserted via the left atrium into the LV cavity. A thin- 
walled latex balloon with a zero pressure filling volume beyond maximum LV lumen capacities was selected for this study to avoid the stiffness of the balloon wall contributing to LV pressure at higher filling volumes. The volume of the balloon wall was assessed with a water-displacement technique, and the same balloon was used throughout each of the studies. A micromanipulator was used to gradually increase LV volumes to values that resulted in no further change in LV developed pressure. LV pressures were determined at as many multiple small increments in volume as were practically possible to improve the accuracy of curve fitting during later analysis. A range of LV pressures and volumes were determined with (-)-isoproterenol HCl (Sigma-Aldrich, South Africa) added to the perfusion buffer $\left(10^{-8} \mathrm{M}\right)$. This approach was employed to assess peak systolic function. Load-independent LV systolic chamber performance was determined from comparisons of the slope of the LV systolic pressure-volume relationship (LV Ees) and load-independent intrinsic systolic myocardial function from the slopes of the LV stress-strain relations (systolic myocardial elastance, En). ${ }^{32}$

\section{Echocardiography}

Left ventricular systolic function was determined in vivo using two-dimensional targeted M-mode echocardiography performed using a 7.5 MHz transducer and a Hewlett Packard Sonos 2500 sector scanner according to the American Society of Echocardiography convention as previously described. ${ }^{31}$ Rats were anesthetized with ketamine and xylazine ${ }^{31}$ and echocardiography was performed blinded, and in random order by a single observer (GRN). Two scans were obtained from each rat and the mean values were calculated. LVEDD and LVESD and posterior wall thickness were measured using the leading edge technique. ${ }^{31}$ These measurements were obtained at baseline and then again 
at regular intervals for 45 minutes after having injected $0.01 \mathrm{mg} / \mathrm{kg}(-)$-isoproterenol $\mathrm{HCl}$ into the peritoneal cavity. This approach was employed to assess systolic functional reserve. Left ventricular systolic chamber function was determined from LV endocardial fractional shortening (LV FSend) and systolic myocardial function from LV midwall fractional shortening (LV FSmid) calculated as previously described. ${ }^{31}$

\section{Blood analysis}

To determine the impact of the model of obesity on circulating insulin, blood glucose, lipids, epinephrine and norepinephrine concentrations and percentage glycosylated haemoglobin (HbA1c), blood samples were obtained from the thoracic cavity immediately after extirpation of the heart. Blood glucose and lipids were determined with a glucometer (Gluco PlusTM; distributed by Cipla Dibcare, Bellville, South Africa) or CardioCheck lipid analyser (CardioCheck ${ }^{\mathrm{TM}}$, Polymer Technology Systems Inc, Indianapolis, USA). Whole blood collected in EDTA tubes was used to determine HbA1c using a Siemens ADVIA 1800, according to the instructions of the manufacturers. Blood samples were collected in serum separation tubes, and centrifuged at $3000 \mathrm{rpm}$ at $4^{\circ} \mathrm{C}$ within 30 minutes of collection. Serum insulin concentrations were determined using a commercially available radioimmunoassay (Coat-A-Count ${ }^{\circledR}$ Insulin, Diagnostic Products Corporation, LA, USA). Epinephrine and norepinephrine concentrations were determined using a commercially available radioimmunoassay (IBL KatCombi, Hamburg, Germany).

\section{Myocardial glycolytic flux rate determinations}

Myocardial glycolytic flux rates were measured in hearts from control and obese rats. To determine the rate of myocardial glycolytic flux, the amount of ${ }^{3} \mathrm{H}_{2} \mathrm{O}$ released from the metabolism of $5-\left[{ }^{3} \mathrm{H}\right]$ glucose by triosephosphate isomerase and enolase steps in the 
glycolytic pathway was measured. Hearts were perfused with $5-\left[{ }^{3} \mathrm{H}\right]$ glucose under normoxic conditions and coronary effluent samples collected for glycolytic flux rate determinations as described previously. ${ }^{23}$

Statistical analysisAnalyses were performed using GraphPad Prism 5.1 Software.

All data are presented as mean \pm standard error of the mean. When comparisons between two groups (diet and control groups) were made, an unpaired Students t-test was performed. Comparisons of the aortic output between the diet and control groups at three different preloads were made using a repeated measures ANOVA followed by the Tukey post hoc test. To compare load independent systolic chamber function between the diet and control groups, the mean slope values in each group were derived from the slopes of linear regressions of the linear portion of the LV systolic pressure-volume curve (end systolic elastance, LV Ees) in individual rats. Comparisons of the mean slope values between the diet and control groups were made using a Students t-test. Comparisons of the aortic output between the diet and control groups with and without FA's at three different preloads were made with a repeated measures ANOVA followed by the Tukey post hoc test. To assess the effects of exposure of the myocardium to an inotropic stimulus on systolic function in the diet and control groups, a repeated measures ANOVA was performed followed by the Tukey post hoc test. 


\section{RESULTS}

\section{Characteristics of the obesity model}

Table 1 shows the impact of the experimental diet on measures of adiposity, ventricular weight, blood biochemistry and systolic blood pressure. Rats fed the experimental diet were approximately $22 \%$ heavier, but had a $100 \%$ larger visceral fat mass than control diet fed rats. The obese rats further had a greater LV weight as compared to control rats. Although fasting blood glucose concentrations and HbAlc levels were similar between the two groups, insulin concentrations tended to be elevated while triglyceride levels were doubled in the obese rats when compared to the control rats. Circulating norepinephrine concentrations were elevated in the obese as compared to the control rats, but tail cuff systolic blood pressures were unchanged.

\section{Myocardial glycolytic flux rates in the presence and absence of insulin}

Myocardial glycolytic flux rates were reduced in obese hearts perfused with glucose alone (Control vs. Obese: no insulin; $0.56 \mathrm{nmol} / \mathrm{g} / \mathrm{min} \pm 0.07 \mathrm{nmol} / \mathrm{g} / \mathrm{min} \quad$ vs. $0.30 \mathrm{nmol} / \mathrm{g} / \mathrm{min} \pm 0.01 \mathrm{nmol} / \mathrm{g} / \mathrm{min}, \mathrm{n}=6-7, \mathrm{p}<0.05)$ and was increased by in vivo insulin concentrations in both groups of hearts (Control vs. Obese: plus insulin; $2.20 \mathrm{nmol} / \mathrm{g} / \mathrm{min}$ $\pm 0.17 \mathrm{nmol} / \mathrm{g} / \mathrm{min}$ vs. $3.68 \mathrm{nmol} / \mathrm{g} / \mathrm{min} \pm 0.28 \mathrm{nmol} / \mathrm{g} / \mathrm{min}, \mathrm{n}=6-7, \mathrm{p}<0.05)$. The insulin induced normalization of the glucose flux rate seen in the obese, insulin resistant hearts was not associated with a matched normalization of the aortic output in these hearts.

\section{Effects of obesity on ex vivo systolic chamber function as assessed in the absence of fatty acids or insulin}

Figure 1 shows the impact of obesity on systolic chamber function as determined using working heart preparations (Figure 1A) and in retrograde perfused, isovolumic, constant 
coronary flow preparations (Figure 1B) in the absence of FA's and insulin. Irrespective of cardiac preloads, hearts from obese rats generated a reduced aortic output compared to that observed in the control rats (Figure 1A). Consistent with data obtained in the working heart preparation, in retrograde perfused, isovolumic, constant coronary flow preparations, hearts from obese rats had a right shift in the LV systolic pressure-volume relationship and a reduced LV Ees (Figure 1B) as compared to that observed in the control rats.

Effects of obesity on ex vivo intrinsic systolic myocardial function in the absence of fatty acids and insulin

The impact of obesity on intrinsic systolic myocardial function as determined in retrograde perfused isovolumic, constant coronary flow heart preparations is demonstrated in Figure 2. Hearts from obese rats displayed a right shift in the LV systolic stress-strain relationship and a reduced load-independent intrinsic systolic myocardial function (LV En) (Figure 2) as compared to that observed in the control rats. Thus, the impact of obesity on systolic chamber function (Figure 1) was in-part attributed to detrimental changes in intrinsic myocardial function.

Effects of exposure of the myocardium to fatty acids or insulin or the combination of

\section{both on ex vivo systolic cardiac function}

The effect of relevant in vivo concentrations of FA's on systolic chamber function can be seen in Figure 3. FA exposure produced a marked inotropic effect in obese rat hearts, but had no effect on control rat hearts. Consequently, in the presence of in vivo concentrations of FA's, the reduced aortic output previously observed in the obese group when perfused with glucose alone (Figure 1A), was normalized as compared to control 
rat hearts. In these un-paced perfusions, heart rates were comparable between the control and obese groups at all preloads (Control vs. Obese: $15 \mathrm{cmH}_{2} \mathrm{O}: 279 \pm 11$ bpm vs. $249 \pm 12$ bpm, $\mathrm{p}=0.10 ; 17.5 \mathrm{cmH}_{2} \mathrm{O}: 284 \pm 11$ bpm vs. $267 \pm 10$ bpm, $\mathrm{p}=0.27 ; 20 \mathrm{cmH}_{2} \mathrm{O}: 286 \pm 12$ bpm vs. $265 \pm 9 \mathrm{bpm}, \mathrm{p}=0.21)$.

The addition of relevant in vivo concentrations of insulin improved aortic output in obese but not control hearts (Figure 3B). These improvements in obese hearts were however small. Aortic outputs in obese, insulin perfused hearts were still lower than for corresponding control, insulin perfused hearts. The combination of in vivo concentrations of FA's with the insulin had no effect on aortic output in either the control of obese rat hearts when compared with FA's alone. With FA's and insulin control and obese hearts had similar aortic outputs at all preloads (Control vs. Obese: $15 \mathrm{cmH}_{2} \mathrm{O}: 44.2 \pm 1.2 \mathrm{ml} / \mathrm{min}$ vs. $46.2 \pm 1.8 \mathrm{ml} / \mathrm{min} ; 17.5 \mathrm{cmH}_{2} \mathrm{O}: 42.9 \pm 1.3 \mathrm{ml} / \mathrm{min} \quad$ vs. $46.2 \pm 1.8 \mathrm{ml} / \mathrm{min} ; 20 \mathrm{cmH}_{2} \mathrm{O}: 48.8 \pm 1.4 \mathrm{ml} / \mathrm{min}$ vs. $48.8 \pm 1.5 \mathrm{ml} / \mathrm{min}, \mathrm{n}=7$ ).

\section{In vivo LV dimensions and systolic chamber and myocardial function}

Table 2 and Figure 4 show the impact of obesity on LV dimensions and systolic chamber (FSend) and myocardial (FSmid) function as determined in vivo using echocardiography. Figure 4 additionally shows the impact of obesity on FSend and FSmid after isoproterenol injection. Obese rat hearts had comparable LVEDD and LVESD, but increased LV posterior wall thickness in comparison to control rats (Table 2). Irrespective of whether systolic function was assessed in the absence (Table 2) or in the presence of a marked inotropic stimulus (Figure 4), hearts from obese rats had a similar isoproterenol-induced increase in systolic function as compared to control rats when exposed to endogenous circulating FA's. 


\section{DISCUSSION}

The main findings of the present study are as follows: In an animal model of obesity and insulin resistance, yet with comparable blood pressure and glycemic indices relative to the control group, we unmasked an inability to maintain LV mechanical function ex vivo when glucose was provided as the sole fuel source. This was evident from reduced LV systolic chamber (reduced aortic outputs over a range of comparable preloads and a reduced LV Ees and LV En) function in the absence of elevated circulating FA and insulin concentrations (but similar glucose concentrations). However, exposure of these hearts to FA concentrations representative of in vivo concentrations of FA's resulted in increased aortic outputs (normalized function relative to controls) as determined over a range of preloads in the obese group. The ability of elevated levels of FA's to normalize systolic function ex vivo in obese rat hearts, may in-part explain the ability of obese rats to generate normal chamber (FSend) and myocardial (FSmid) systolic function in vivo at baseline and in the presence of a marked inotropic stimulus.

The healthy heart is thought to be metabolically flexible, in that it can effectively utilize various substrates in order to maintain LV function. This has been shown to be true when perfusing isolated hearts from healthy animals with either glucose alone, or in combination with FA in the present study and those of others. ${ }^{33}$ However, the hearts ability to effectively use glucose as an energy source is compromised in obese insulin resistant states, ${ }^{34}$ which, as we demonstrate, translates into reduced intrinsic systolic myocardial function and LV chamber function when the heart is forced to rely solely on 
glucose as a fuel source. This observation is in line with a number of studies reporting reduced contractile function in isolated cardiomyocytes or ex vivo perfused hearts obtained from obese animals where glucose was provided as the sole substrate. ${ }^{12,18,35-37}$

Our data show that although the addition of insulin improves cardiac function in our obese insulin resistant animals, the mechanical function is still significantly compromised when compared to control animals. We however demonstrate that with the inclusion of in vivo representative levels of FA's to the perfusate, hearts from obese rats developed an increased pump function to levels commensurate with control values at all the preloads assessed. It is unlikely that augmented loading conditions may account for these observations in the working heart perfusion model since preload, afterload and heart rate were comparable between the FA perfused hearts from the control and obese animals. This observation may therefore in part explain the reduced contractile function noted in the previously mentioned studies, ${ }^{12,18,35-37}$ as well as the comparable myocardial function noted in obese insulin resistant rats relative to controls in vivo during resting conditions and following inotropic stimulation (present study). Surprisingly, the combined inclusion of both insulin and FA's in the perfusion buffer in obese hearts did not have an additive effect. Systolic function (aortic output) remaining unchanged when compared to the hearts treated with FA's only. The myocardial dependence on elevated levels of FA's as a fuel to maintain LV systolic function in obese rats is in keeping with the preserved in vivo LV systolic function noted in other diet induced models of obesity. ${ }^{13,19}$ Our results thus emphasize the importance of including FA as a fuel source when assessing ex vivo contractile parameters in models of obesity and insulin resistance. 
Interestingly, the normal LV pump function noted in obese mice relative to controls in the study of Yan et $\mathrm{al}^{17}$ occurred in the absence of elevated circulating FA levels, a finding that differs from the model used in the present study where circulating FA's were previously reported to be elevated. ${ }^{23}$ Thus it is possible that in obesity and insulin resistance, the presence of FA's per se and possibly the heart's ability to use this fuel source effectively may be equally important in the maintenance of LV pump function. We therefore speculate that during the myocardial metabolic adaptation phase of obesity, prior to the development of frank diabetes and hypertension, energy derived from FA metabolism plays a much more important role in maintaining LV pump function and functional reserve than in healthy hearts from lean controls.

Although we did not investigate substrate metabolism or identify the expression of genes involved in glucose and fatty acid metabolism in detail, it is clear from our glycolytic flux rate determinations that in the very early stages of diet induced obesity, cardiomyocyte glycolysis and glucose oxidation rates are decreased. This is possibly due to a reduction in GLUT4 expression and attenuated insulin stimulated GLUT4 translocation which may lead to increased FA oxidation and an increase in myocardial oxygen consumption. ${ }^{38}$ These changes occur prior to increased expression of peroxisome proliferator activated receptor-alpha target genes. ${ }^{38}$ It is reasonable to believe that this change in substrate flexibility is inevitably aimed at maintaining adequate myocardial energy production to preserve LV pump function although it may come at the expense of reduced cardiac efficiency. Importantly, these changes have been shown to occur in the obese insulin 
resistant animals prior to the development of diabetes associated systolic dysfunction. ${ }^{34}$ Despite the important role of elevated FA's on myocardial function in obesity it is further clear that a persistent increase in circulating FA levels is associated with adverse myocardial consequences such as atrial fibrillation ${ }^{39}$ and diastolic dysfunction ${ }^{40}$ whereas increased myocardial FA uptake and the consequent storage thereof within heart tissue are associated with reduced LV systolic function in humans with impaired glucose tolerance. ${ }^{41}$ In line with this observation, Peterson et $\mathrm{al}^{42}$ has also speculated that the altered myocardial substrate utilization profile associated with obesity and insulin resistance may contribute to cardiac dysfunction. It may therefore be speculated that detrimental consequences of increased FA supply to the myocardium are only seen when increased FA supply exceeds the hearts ability to oxidize the $\mathrm{FA}^{43}$ which in turn may lead to the formation of toxic lipid intermediates. ${ }^{44,45}$ Alternatively, long term exposure of the heart to high levels of a particular substrate may render the metabolic network of the heart incapable of adapting to a superimposing metabolic stress, consequently resulting in cardiac dysfunction. ${ }^{17}$ Although it may seem reasonable to attempt to modify myocardial substrate metabolism by inhibiting FA oxidation and increasing glucose oxidation, Yan et $\mathrm{al}^{17}$ demonstrated that this could also be disadvantageous to the heart in the setting of obesity, therefore suggesting that this approach should be pursued with caution. This notion is supported by the present study as the importance of FA's as a fuel source for the heart in the obese insulin resistant condition is clearly demonstrated. The complicated balance between FA availability and cardiac function was further demonstrated by various authors where an acute to short term reduction in circulating FA levels 
significantly reduced cardiac function in patients with heart failure and healthy participants respectively. ${ }^{46,47}$

The increased systolic functional response to FA's in obese, but not control rat hearts may not be the only explanation for the preserved pump function noted in vivo in the present study. Elevated circulating norepinephrine concentrations have been noted in obese humans ${ }^{48}$ and also in obese rats in the present study, which may enhance pump function in vivo. These elevated norepinephrine levels may not only have direct inotropic effects but potentially also contribute to increased lipolysis, ${ }^{49}$ fatty acid availability and $\beta$-oxidation ${ }^{50}$ which potentially also contributes to the significant improvement in systolic function seen with the inclusion of FA's.

Although myocardial contractile dysfunction may be due to both diastolic and systolic dysfunction, we did not assess diastolic function in the hearts in this study. This aspect of cardiac dysfunction more difficult to assess and fell beyond the scope of this study. While we did not report data documenting in vivo free FA levels in this study, we have previously demonstrated that rats fed this high caloric diet for 16 weeks have circulating free FA levels that are double that of the control group. ${ }^{23}$ This study importantly used relevant in vivo concentrations of FA for control and obese animals when assessing their impact on cardiac pump function. In addition comparisons between in vivo and ex vivo functional parameters utilized in this study provide a more comprehensive assessment of cardiac systolic function in these hearts. 
In conclusion, the present study indicates that cardiac systolic dysfunction occurs in obesity with insulin-resistance in the absence of FA and insulin. However in the presence of normally elevated in vivo concentrations of FA's, cardiac systolic function is preserved in the isolated heart thus mimicking in vivo myocardial pump function. The study thus demonstrates the importance of free FA's in the maintenance of cardiac systolic function at rest and in response to inotropic stimulation in obesity and insulin resistance. 


\section{Disclosure}

No conflicts of interest are declared by the authors. All authors have approved the final article.

\section{Author Contributions}

Writing the paper and scientific input towards the paper: WS, EFdT, GRN, AJW, AL; Experimental work: EFdT, GRN, WS, AJW; Statistical analysis: WS, EFdT, AJW.

\section{Funding}

This study was supported by funding from the Circulatory Disorders Research Fund (CDRF) and the South African National Research Foundation. 


\section{REFERENCES}

1. Kenchaiah S, Evans JC, Levy D, Wilson PW, Benjamin EJ, Larson MG et al. Obesity and the risk of heart failure. New Engl J Med 2002;347:305-313 doi:10.1056/NEJMoa020245

2. Berkalp B, Cesur V, Corapcioglu D, Erol C, Baskal N. Obesity and left ventricular diastolic dysfunction. Int $\mathrm{J}$ Cardiol 1995;52:23-26 doi:10.1016/0167$5273(95) 02431-\mathrm{U}$

3. Pascual M, Pascual DA, Soria F, Vicente T, Hernandez AM, Tebar FJ et al. Effects of isolated obesity on systolic and diastolic left ventricular function. Heart 2003;89:1152-1156 dio:10.1136/heart.89.10.1152

4. Zarich SW, Kowalchuk GJ, McGuire MP, Benotti PN, Mascioli EA, Nesto RW. Left ventricular filling abnormalities in asymptomatic morbid obesity. Am J Cardiol 1991;68:377-381

5. Stoddard MF, Tseuda K, Thomas M, Dillon S, Kupersmith J. The influence of obesity on left ventricular filling and systolic function. Am Heart J 1992;124:694699 doi:10.1016/0002-8703(92)90280-9

6. De Simone G, Deveroux RB, Mureddu GF, Roman MJ, Ganau A, Alderman MH et al. Influence of obesity on left ventricular midwall mechanics in arterial hypertension. Hypertension 1996;28:276-283 doi:10.1161/01.HYP.28.2.276

7. Mureddu GF, de Simone G, Greco R, Rosato GF, Contaldo F. Left ventricular filling pattern in uncomplicated obesity. Am J Cardiol 1996;77:509-514. dio:10.1016/S0002-9149(97)89346-0 
8. Otto ME, Belohlavek M, Khandheria B, Gilman G, Svatikova A, Somers V. Comparison of right and left ventricular function in obese and nonobese men. Am J Cardiol 2004;93:1569-1572 dio:10.1016/j.amjcard.2004.02.073

9. Saglione R, Dichiara MA, Indovina A, Lipari R, Ganquzza A, Parinello G et al. Left ventricular diastolic and systolic function in normotensive obese subjects: influence of degree and duration of obesity. Eur Heart J 1992;13:738-742 doi:738742

10. Peterson LR, Waggoner AD, Schechtmann KB, Meyer T, Gropler RJ, Barzilia B et al. Alterations in left ventricular structure and function in young healthy obese women: assessment by echocardiography and tissue Doppler imaging. J Am Coll Cardiol 2004;43:1399-1404 doi:10.1016/j.jacc.2003.10.062

11. Wong CY, O’Moore-Sullivan $\mathrm{T}$, Leano R, Byrne N, Beller E, Marwick TH. Alterations of left ventricular myocardial characteristics associated with obesity. Circulation 2004;110:3081-3087 doi:10.1161/01.CIR.0000147184.13872.0F

12. Du Toit EF, Nabben M, Lochner A. A potential role for Angiotensin II in obesity induced cardiac hypertrophy and ischaemic/reperfusion injury. Basic Res Cardiol 2005;100:346-354 doi:10.1007/s00395-005-0528-5

13. Carroll JF, Zenebe WJ, Strange, TB. Cardiovascular function in a rat model of diet induced obesity Hypertension $2006 ; 48: 65-72$ doi:10.1161/01.HYP.0000224147.01024.77

14. Ouwens DM, Diamant M, Fodor M, Habets DD, Pelsers MM, El Hasnaoui M et al. Cardiac contractile dysfunction in insulin-resistant rats fed a high-fat diet is 
associated with elevated CD36-mediated fatty acid uptake and esterification. Diabetologia 2007;50:1938-1948 dio:10.1007/s00125-007-0735-8

15. Wilson CR, Tran MK, Salazar KL, Young ME, Taegtmeyer H. Western diet, but not high fat diet, causes derangements of fatty acid metabolism and contractile dysfunction in the heart of Wistar rats. Biochem J 2007;406:457-467 doi:10.1042/BJ20070392

16. Aasum E, Khalid AM, Gudbrandsen OA, How OJ, Berge RK, Larsen TS. Fenofibrate modulates cardiac and hepatic metabolism and increases ischemic tolerance in diet induced obese mice. J Mol Cell Cardiol 2008;44:201-209 doi:10.1016/j.yjmcc.2007.08.020

17. Yan J, Young ME, Cui L, Lopaschuk GD, Liao R, Tian R. Increased glucose uptake and oxidation in mouse hearts prevent high fatty acid oxidation but cause cardiac dysfunction in diet-induced obesity. Circulation 2009;119:2818-2828 doi:10.1161/CIRCULATIONAHA.108.832915

18. Nduhirabandi F, Du Toit EF, Blackhurst D, Marais D, Lochner A. Chronic melatonin consumption prevents obesity-related metabolic abnormalities and protects the heart against myocardial ischemia and reperfusion injury in a prediabetic model of diet-induced obesity. J Pineal Res 2011;50:171-182. dio:10.1111/j.1600-079X.2010.00826.x

19. Wensley I, Salaveria K, Bulmer AC, Donner DG, du Toit EF. Myocardial structure, function and ischaemic tolerance in a rodent model of obesity with insulin resistance. Exp Physiol 2013;98:1552-1564 doi:10.1113/expphysiol.2013.074948 
20. Alpert MA, Lambert CR, Panayiotou H, Terry BE, Cohen MV, Massey CV et al. Relation of duration of morbid obesity to left ventricular mass, systolic function, and diastolic filling, and effect of weight loss. Am J Cardiol 1995;76:1194-1197 doi:10.1016/S0002-9149(99)80338-5

21. Koutsari C, Jensen MD. Thematic review series: patient-oriented research. Free fatty acid metabolism in human obesity. J Lipid Res 2006;47:1643-1650 doi:10.1194/jlr.R600011-JLR200

22. Mazumder PK, O’Neill BT, Roberts MW, Buchanan J, Yun UJ, Cooksey RC et al. Impaired cardiac efficency and increased fatty acid oxidation in insulin-resistant ob/ob mouse hearts. Diabetes 2004;53:2366-2374 dio:10.2337/diabetes.53.9.2366

23. Du Toit EF, Smith W, Muller C, Strijdom H, Stouthammer B, Woodiwiss AJ et al. Myocardial susceptibility to ischemic-reperfusion injury in a prediabetic model of dietary-induced obesity. Am J Physiol 2008;294:H2336-H2343 doi:10.1152/ajpheart.00481.2007

24. Salie R, Huisamen B, Lochner A. High carbohydrate and high fat diets protect the heart against ischaemia/reperfusion injury. Cardiovasc Diabetol 2014;13:109. doi: 10.1186/s 12933-014-0109-8.

25. Pickavance L, Tadayyon M, Widdowson PS, Buckingham RE, Wilding JP. Therapeutic index for rosiglitazone in dietary obese rats: separation of efficacy and haemodilution. Brit J Pharmacol 1999;128:1570-1576 doi:10.1038/sj.bjp.0702932

26. Huisamen B, Genis A, Marais E, Lochner A. Pre-treatment with a DPP-4 inhibitor is infarct sparing in hearts from obese, pre-diabetic rats. Cardiovasc Drugs Ther 2011;25:13-20 doi:10.1007/s10557-010-6271-7 
27. Buñag RD. Validation in awake rats of a tail-cuff method for measuring systolic pressure. J Appl Physiol 1973;34:279-282

28. Opie LH, Mansford KR, Owen P. Effects of increased heart work on glycolysis and adenine nucleotides in the perfused heart of normal and diabetic rats. Biochem $\mathrm{J}$ $1971 ; 124: 475-490$.

29. Woodiwiss AJ, Tsotetsi OJ, Sprott S, Lancaster EJ, Mela T, Chung ES et al. Reduction in myocardial collagen cross-linking parallels left ventricular dilatation in rat models of systolic chamber dysfunction. Circulation 2001;103:155-160 doi:10.1161/01.CIR.103.1.155

30. Lopaschuk GD, Barr RL. Measurement of fatty acid and carbohydrate metabolism in the isolated working rat heart. Mol Cell Biochem 1997;172:137-147 doi: $10.1007 / 978-1-4615-6353-2 \_15$

31. Norton GR, Woodiwiss AJ, Gaasch WH, Mela T, Chung ES, Aurigemma GP et al. Heart failure in pressure overload hypertrophy: the relative roles of ventricular remodeling and myocardial dysfunction. J Am Coll Cardiol 2002;39:664-671 dio:10.1016/S0735-1097(01)01792-2

32. Weber KT, Janicki JS, Shroff SG, Pick R, Chen RM, Bashey RI. Collagen remodeling of the pressure-overloaded, hypertrophied nonhuman primate myocardium. Circ Res 1988;62(4):757-65.

33. Buchanan J, Mazumder PK, Hu P, Chakrabarti G, Roberts MW, Yun UJ et al. Reduced cardiac efficiency and altered substrate metabolism precedes the onset of hyperglycemia and contractile dysfunction in two mouse models of insulin 
resistance and obesity. Endocrinology 2005;146:5341-5349 doi:10.1210/en.20050938.

34. Liu Q, Docherty JC, Rendell JC, Clanachan AS, Lopaschuk GD. High levels of fatty acids delay the recovery of intracellular $\mathrm{pH}$ and cardiac efficiency in postischemic hearts by inhibiting glucose oxidation. J Am Coll Cardiol 2002;39:718725 doi:10.1016/S0735-1097(01)01803-4

35. Ren J, Sowers JR, Walsh MF, Brown RA. Reduced contractile response to insulin and IGF-I in ventricular myocytes from genetically obese Zucker rats. Am J Physiol 2000;279:H1708-H1714.

36. Relling DP, Esberg LB, Fang CX, Johnson WT, Murphy EJ, Carlson EC et al. High-fat diet induced juvenile obesity leads to cardiomyocyte dysfunction and upregulation of Foxo3a transcription factor independent of lipotoxicity and apoptosis. J Hypertens 2006;24:549-561 doi:10.1097/01.hjh.0000203846.34314.94

37. Ren J, Dong F, Cai GJ, Zhao P, Nunn JM, Wold LE et al. Interaction between age and obesity on cardiomyocyte contractile function: role of leptin and stress signaling. PLoS One 2010;5:e10085 doi: 10.1371/journal.pone.0010085

38. Wright JJ, Kim J, Buchanan J, Boudina S, Sena S, Bakirtzi K,et al. Mechanisms for increased myocardial fatty acid utilization following short-term high-fat feeding. Cardiovasc Res. 2009 May 1;82(2):351-60. doi: 10.1093/cvr/cvp017.

39. Khawaja O, Bartz TM, Ix JH, Heckbert SR, Kizer JR, Zieman SJ et al. Plasma free fatty acids and risk of atrial fibrillation (from the Cardiovascular Health Study). Am J Cardiol 2012;110:212-216 doi:10.1016/j.amjcard.2012.03.010 
40. Leichman JG, Aguilar D, King TM, Vlada A, Reyes M, Taegtmeyer H. Association of plasma free fatty acids and left ventricular diastolic function in patients with clinically severe obesity

41. Labbé SM, Grenier-Larouche T, Noll C, Phoenix S, Guérin B, Turcotte EE et al. Increased myocardial uptake of dietary fatty acids linked to cardiac dysfunction in glucose-intolerant humans. Diabetes 2012;61:2701-2710 doi:10.2337/db11-1805.

42. Peterson LR, Herrero P, Schechtman KB, Racette SB, Waggoner AD, KisrievaWare $\mathrm{Z}$ et al. Effect of obesity and insulin resistance on myocardial substrate metabolism and efficiency in young women. Circulation 2004;109:2191-2196 doi:10.1161/01.CIR.0000127959.28627.F8

43. Young ME, Guthrie PH, Razeghi P, Leighton B, Abbasi S, Patil S, et al. Impaired long-chain fatty acid oxidation and contractile dysfunction in the obese Zucker rat heart. Diabetes 2002;51:2587-2595 doi:10.2337/diabetes.51.8.2587

44. Zhou YT, Grayburn P, Karim A, Shimabukuro M, Higa M, Baetens D et al. Lipotoxic heart disease in obese rats: implications for human obesity. Proc Natl Acad Sci USA 2000;97:1784-1789 doi:10.1073/pnas.97.4.1784

45. Chess DJ, Stanley WC. Role of diet and fuel overabundance in the development and progression of heart failure. Cardiovasc Res 2008;79:269-278 doi:10.1093/cvr/cvn074

46. Lehto HR, Pärkkä J, Borra R, Tuunanen H, Lepom Am J Clin Nutr. 2006 Aug;84(2):336-41.aki V, Parkkola R, Knuuti J, Nuutila P, Iozzo P. Effects of acute and one-week fatty acid lowering on cardiac function and insulin sensitivity in 
relation with myocardial and muscle fat and adiponectin levels. J Clin Endocrinol Metab. 2012 Sep;97(9):3277-84. doi: 10.1210/jc.2012-1219.

47. Salerno A, Fragasso G, Esposito A, Canu T, Lattuada G, Manzoni Get al. Effects of short-term manipulation of serum FFA concentrations on left ventricular energy metabolism and function in patients with heart failure: no association with circulating bio-markers of inflammation. Acta Diabetol. 2015;52:753-761.

48. Egan BM, Schork NJ, Weder AB. Regional hemodynamic abnormalities in overweight men. Focus on alpha-adrenergic vascular responses. Am J Hypertens 1989;2:428-434. doi: 10.1093/ajh/2.6.428.

49. Arner P. Catecholamine-induced lipolysis in obesity. Int J Obes Relat Metab Disord. 1999;23:10-13.

50. Lopaschuk GD, Ussher JR, Folmes CDL, Jaswal JS and Stanley WC. Myocardial fatty acid metabolism in health and disease. Physiol Rev. 2010;90:207-258. 


\section{Legends.}

Figure 1. Impact of obesity on ex vivo left ventricular (LV) systolic chamber function in the absence of exposure of the myocardium to fatty acids in the coronary perfusate. Panel A illustrates aortic outputs at incremental filling pressures in working heart preparations, and panel B shows LV systolic pressures over a range of filling volumes in isovolumic retrograde perfused hearts. Comparison of the slopes of these relationships (LV Ees) are shown in the insert to panel B.

$* \mathrm{p}<0.05$ versus controls.

Figure 2. Impact of obesity on ex vivo left ventricular (LV) intrinsic myocardial systolic function, as assessed from LV systolic stress-strain relations in isovolumic heart preparations in the absence of exposure of the hearts to fatty acids in the coronary perfusate. Comparisons of the slopes of these relations (LV En) are shown in the insert. $* \mathrm{p}<0.05$ versus controls.

Figure 3. A. Impact of obesity on ex vivo left ventricular (LV) systolic chamber function in the absence as compared to the presence of fatty acids in the coronary perfusate.

* $p<0.05$ versus controls and diet $+F A$. B. Impact of obesity on ex vivo left ventricular (LV) systolic chamber function in the absence as compared to the presence of insulin in the coronary perfusate. ${ }^{*} \mathrm{p}<0.05$ versus controls. $\dagger \mathrm{p}<0.05$ vs. control + insulin

Figure 4. Impact of obesity on left ventricular systolic chamber (endocardial fractional shortening [FS]) (panel A) and myocardial (midwall FS) (panel B) function at baseline and after an intraperitoneal injection of (-)-isoproterenol $\mathrm{HCl}(0.01 \mathrm{mg} / \mathrm{kg})$. 
$* \mathrm{p}<0.001$ versus baseline. 